\title{
THE NEAR INFRARED FEH LINES AS INDICATORS OF SURFACE GRAVITY OF M STARS
}

\author{
RICARDO PIORNO SCHIAVON AND BEATRIZ BARBUY \\ Instituto Astronômico Geofísico - USP \\ CP 9638, São Paulo, 01065-970, SP, Brazil \\ ripisc@astro1.iagusp.usp.br, barbuy@vax.iagusp.usp.br
}

We compute synthetic spectra in the region around $1 \mu \mathrm{m}$, including the Wing-Ford band (WFB) of Iron Hydride (FeH) in the calculations. This band is known to be a good indicator of surface gravities of $M$ stars. Employing Kurucz model atmospheres, we study the response of the intensity of the WFB to atmospheric parameters and check our results against observations of $M$ dwarfs. This study is part of an ongoing project which aims to investigate the $\mathrm{M}$ dwarf-to-giant ratio in galaxies, through a population synthesis method, exploring a number of spectral indicators in the near infrared, such as the WFB, the NaI, CaII and CO near infrared features.
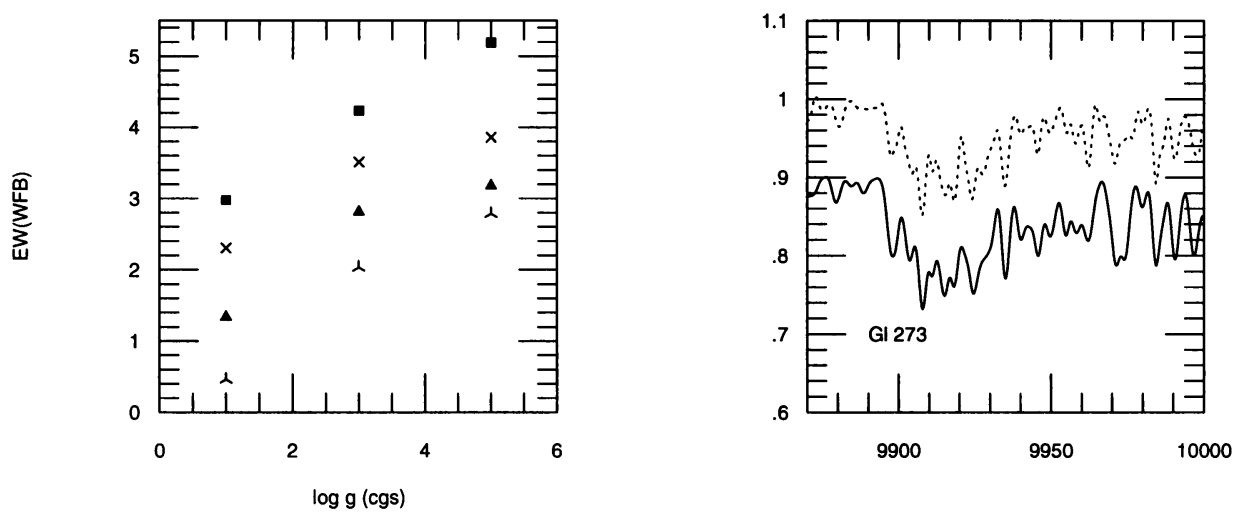

Figure 1. Left: the behaviour of the Equivalent Width of $\mathrm{FeH}$ lines in the spectral interval 9890-9970 $\AA$ as a function of stellar surface gravity $(\log g)$ for effective temperatures of Teff $=3500,3700,3900$ and $4200 \mathrm{~K}$. Right: comparison between synthetic and observed spectra of an $M$ dwarf star of $(\mathrm{Teff}, \log \mathrm{g},[\mathrm{Fe} / \mathrm{H}])=3500,5.1,0.0$. This work was based upon observations collected at Laboratório Nacional de Astrofísica, Brazópolis, Brazil. 